\title{
Glassy dynamics, metastability limit and crystal growth in a lattice spin model
}

\author{
Andrea Cavagna, Irene Giardina, Tomas Grigera \\ Center for Statistical Mechanics and Complexity, INFM Roma "La Sapienza" and \\ Dipartimento di Fisica, Università di Roma "La Sapienza", 00185 Roma, Italy
}

(Dated: July 5, 2002)

\begin{abstract}
We introduce a lattice spin model where frustration is due to multibody interactions rather than quenched disorder in the Hamiltonian. The system has a crystalline ground state and below the melting temperature displays a dynamic behaviour typical of fragile glasses. However, the supercooled phase loses stability at an effective spinodal temperature, and thanks to this the Kauzmann paradox is resolved. Below the spinodal the system enters an off-equilibrium regime corresponding to fast crystal nucleation followed by slow activated crystal growth. In this phase and in a time region which is longer the lower the temperature we observe a violation of the fluctuation-dissipation theorem analogous to structural glasses. Moreover, we show that in this system there is no qualitative difference between a locally stable glassy configuration and a highly disordered polycrystal.
\end{abstract}

When cooled fast enough, most liquids can be brought below their melting temperature $T_{m}$ while avoiding crystallization. In this supercooled phase the viscosity increases with decreasing temperature, until at the glass transition $T_{g}$ relaxation time becomes too long and equilibration cannot be achieved within experimentally accessible times. In 1948, W. Kauzmann [i] noted that if the entropy of a supercooled liquid is extrapolated below $T_{g}$, it becomes equal to the crystal entropy at a temperature $T_{s}>0$, and even smaller than zero if extrapolated further. This entropy crisis is never actually observed, because the glass transition intervenes before. However, Kauzmann found it paradoxical that it was just a kinetic phenomenon (the glass transition) that saved the liquid from a thermodynamic nonsense.

In the context of the Adams-Gibbs theory [2, the entropy crisis has however an interpretation: the entropy difference between crystal and liquid is related to the configurational entropy $\Sigma$, that is the entropic contribution due to the presence of an exponentially high number of different glassy minima. The vanishing of $\Sigma$ at $T_{s}$ signals a thermodynamic transition to a new phase, characterized by a sub-exponential number of glassy states, separated by infinite free-energy barriers. This picture is exact for some mean-field spin-glass systems [3], and it may be the correct resolution of the Kauzmann paradox even for real structural glasses.

Despite analytic and numerical work supporting the entropy crisis scenario 堛, there is another way to avoid the Kauzmann paradox, which, interestingly enough, was proposed by Kauzmann himself [1]. He rejected the idea of a thermodynamic glassy phase, and of a transition at $T_{s}$. What Kauzmann hypothesized is the existence of a metastability limit of the supercooled liquid phase, below which crystal nucleation becomes faster than liquid equilibration. More precisely, he defined an effective spinodal temperature $T_{\mathrm{sp}}>T_{s}$ below which "the free energy barrier to crystal nucleation becomes reduced to the same height as the barrier to simpler motions". Below $T_{\mathrm{sp}}$ the supercooled liquid is operationally meaningless and thus the paradox is avoided. However, the metastability limit may prove impossible to observe experimentally if the equilibration time at $T_{\mathrm{sp}}$ is much larger than the experimental time, that is if $T_{\mathrm{sp}}<T_{g}$.

In the Kauzmann scenario, the off-equilibrium phase below $T_{\mathrm{sp}}$ basically consists in a very slow crystal domain growth. This suggests two criteria to detect whether a system has such a metastability limit or not, even when $T_{\mathrm{sp}}$ is experimentally inaccessible. First, we may think that there is a qualitative difference between a disordered glassy configuration, obtained by quenching a liquid, and a polycrystalline configuration, however rich in defects this is, and however slow crystal growth may be. Secondly, we know that the off-equilibrium dynamics of simple domain growth (as in the Ising model) can be distinguished from glassy dynamics (as observed in structural glasses) by a different violation of the fluctuationdissipation theorem (FDT) [5, 6].

In this Letter we present a model where the Kauzmann paradox is avoided by a metastability limit. The model is thus a good test for the two criteria discussed above. We shall find that neither criterion is sharp enough to discriminate such a system from a typical structural glass. More precisely, if our experimental time were not long enough to explicitly observe the loss of stability of the liquid at $T_{\mathrm{sp}}$, it would be impossible to distinguish the present system from an ordinary fragile glass. The reason is that below $T_{\mathrm{sp}}$ crystal nucleation is fast, but crystal growth becomes very slow, with many crystal droplets trying to expand in a liquid background [7]. In such a situation distinguishing between a truly disordered glass and a mixture of tiny mismatched crystallites becomes very hard, and FDT violation is nontrivial.

The aim of our study is twofold. First, we want to show that Kauzmann's resolution of the Kauzmann paradox is valid at least in one simple system, and that it is not necessarily in conflict with glassy phenomenology. Second, we hope this example will help to develop some more strict criteria to distinguish systems with a thermodynamic transition at $T_{s}$, from those where the Kauzmann 
scenario holds. The Hamiltonian of our model is,

$$
H=\sum_{i=1}^{N}\left(1+s_{i}\right) f_{i}, \quad f_{i}=s_{i}^{W} s_{i}^{S} s_{i}^{E} s_{i}^{N},
$$

where $W$ is for west, $S$ for south, etc. The spins $s_{i}= \pm 1$ belong to a two-dimensional square lattice of linear size $L$. We perform single-spin-flip Monte Carlo simulations in square lattices with $L=100$ and $L=500$ [8].

The disordered version of this model was first introduced in [9] to describe an ensemble of coupled two level systems, whence its name, CTLS. There has been recently much interest in lattice models of this sort, where multibody interactions in the Hamiltonian ensure frustration even without quenched disorder [10, 11, 12, 13.

The CTLS has a crystalline ground state obtained by covering the lattice with the following non-overlapping 5 spins elements: $s_{i}=-1, s_{i}^{W}=s_{i}^{S}=s_{i}^{E}=s_{i}^{N}=+1$. The ground state energy density is $e_{\mathrm{GS}}=-1.6$. The unit cell size is $5 \times 5$, and this together with the symmetry $x \rightarrow \mp x, y \rightarrow \pm y$, gives a ground state degeneracy of 50. The finite degeneracy of the crystal is a key feature of the CTLS compared to the plaquette model of [11, since it will allow us to directly measure the amount of crystalline order in the system.

Model (1) has a first order (melting) transition. To locate the melting temperature $T_{m}$ we compute the free energy $f$ for the crystal (CR) and liquid (LQ) and ask that $f_{\mathrm{CR}}\left(T_{m}\right)=f_{\mathrm{LQ}}\left(T_{m}\right)$. The free energy is obtained integrating the energy $(\beta=1 / T): \beta f(\beta)=\beta_{0} f\left(\beta_{0}\right)+$ $\int_{\beta_{0}}^{\beta} d \beta^{\prime} e\left(\beta^{\prime}\right)$, taking $\beta_{0}=0$ for the liquid, and $\beta_{0}=\infty$ for the crystal. The equilibrium energies are well fitted by,

$$
\begin{aligned}
& e_{\mathrm{LQ}}(T)=-1.8 \tanh (1.1 / T), \\
& e_{\mathrm{CR}}(T)=e_{\mathrm{GS}}+5.5 \times 10^{-5} T^{13} .
\end{aligned}
$$

Using these relations we find $T_{m}=1.30$. Unless cooling is exceedingly slow, crystallization is not attained at $T_{m}$, and the liquid can be kept at equilibrium in its supercooled phase $T<T_{m}$. Extrapolation of $e_{\mathrm{LQ}}(T)$ and $f_{\mathrm{LQ}}(T)$ gives the temperature $T_{s}$ where the entropy of the supercooled liquid equalizes that of the crystal, namely the Kauzmann paradox temperature. We find $T_{s}=0.91$.

The equilibrium dynamics of the CTLS in the supercooled phase can be studied by measuring the normalized spin-spin correlation function, $C_{n}\left(t, t_{w}\right)=\left[\left\langle s\left(t_{w}\right) s(t)\right\rangle-\right.$ $\left.\langle s\rangle^{2}\right] /\left[1-\langle s\rangle^{2}\right]$, with $t>t_{w}$. In equilibrium $C_{n}\left(t, t_{w}\right)=$ $C_{n}\left(t-t_{w}\right)$, and we find that the correlation can be fitted to a stretched exponential, $C_{n}=\exp \left[-\left(t-t_{w} / \tau\right)^{\beta}\right]$. In Fig. 11 we plot the relaxation time $\tau$ as a function of $T$, together with a power law fit $\tau=A /\left(T-T_{c}\right)^{\gamma}$, with $T_{c}=1.06, \gamma=2.29$. The accuracy of the fit suggests that the CTLS is a fragile system, a fact supported by the Angell plot 14 in the inset of Fig. 1, which compares the relaxation times of the CTLS and a strong system, the two-dimensional plaquette model (2d-PQ)

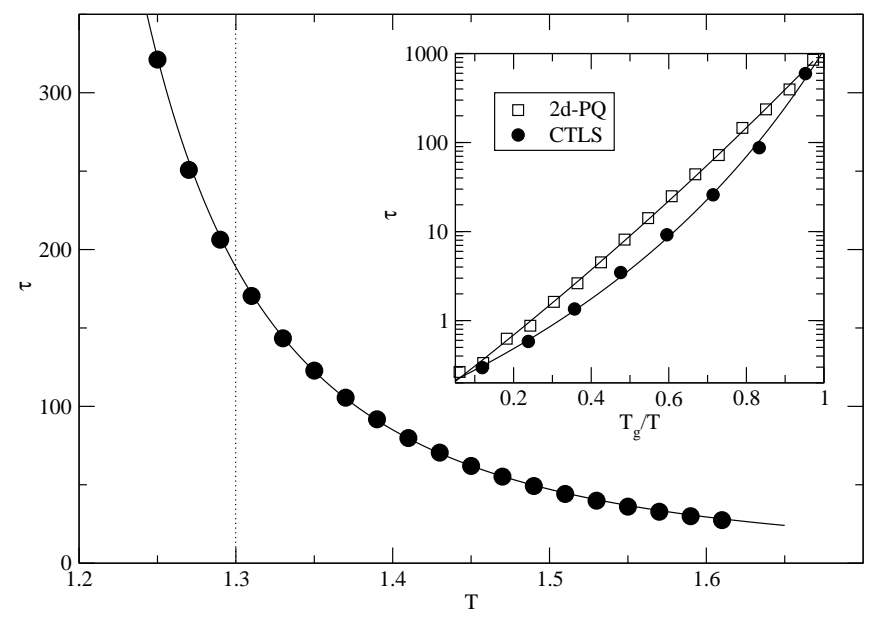

FIG. 1: Relaxation time as a function of the temperature. Full line: power law fit. Inset: fragility plot for a comparison of the CTLS with the model studied in [12]. $T_{g}$ is an operational 'glass transition', defined by $\tau\left(T_{g}\right)=1000$. $L=500$.

studied in 12]. Data are fitted to a Vogel-Fulcher form, $\tau=\tau_{0} \exp \left[\Delta /\left(T-T_{0}\right)\right]$. In the CTLS we find $T_{0}=0.76$ for $T \in[1.2: 1.7]$, and $T_{0}=0.90$ for $T \in[1.2: 1.4]$.

Below about $T \approx 1.2$ the relaxation time cannot be measured, because crystal nucleation starts before the liquid equilibrates. This can be seen in Fig. 2, where we plot the energy density vs. time at four different temperatures below $T_{m}$, with random initial condition. At $T=1.26$, the system relaxes in the supercooled liquid and remains in this phase up to our experimental time, $2 \times 10^{6} \mathrm{MCS}$. On the other hand, for $T=1.23$, after initial equilibration in the liquid phase, the system makes a sharp transition to the crystal: on average crystal nucleation starts at about $10^{5} \mathrm{MCS}$, while crystal growth is completed in $10^{6}$ MCS. At $T=1.18$, however, nucleation starts roughly after $10^{4} \mathrm{MCS}$, while complete crystallization is achieved in more than $10^{6}$ MCS. At $T=1$, the liquid plateau disappears and crystallization is still incomplete after the largest time. Thus at lower temperatures nucleation is faster, but crystal growth is slower.

To pinpoint the liquid metastability limit we use standard nucleation theory 15. A necessary condition for the existence of the supercooled liquid is that the crystal nucleation time $\tau_{n u c}$ is much longer than the liquid equilibration time $\tau_{e q}$. We can estimate $\tau_{n u c}(T)$ close to a reference temperature $T^{\star}$, assuming that the surface tension is constant. We have,

$$
\tau_{n u c}(T)=\exp \left\{\frac{T^{\star} \delta f\left(T^{\star}\right)}{T \delta f(T)} \log \left[\tau_{n u c}\left(T^{\star}\right)\right]\right\},
$$

where $\delta f(T)=f_{\mathrm{LQ}}(T)-f_{\mathrm{CR}}(T)$ is the bulk free energy difference between supercooled liquid and crystal. We have chosen $T^{\star}=1.23$. To estimate the liquid equilibration time we note that the correlation $C(t)$ drops to zero in about 20 relaxation times, and thus $\tau_{e q}=20 \tau$. 


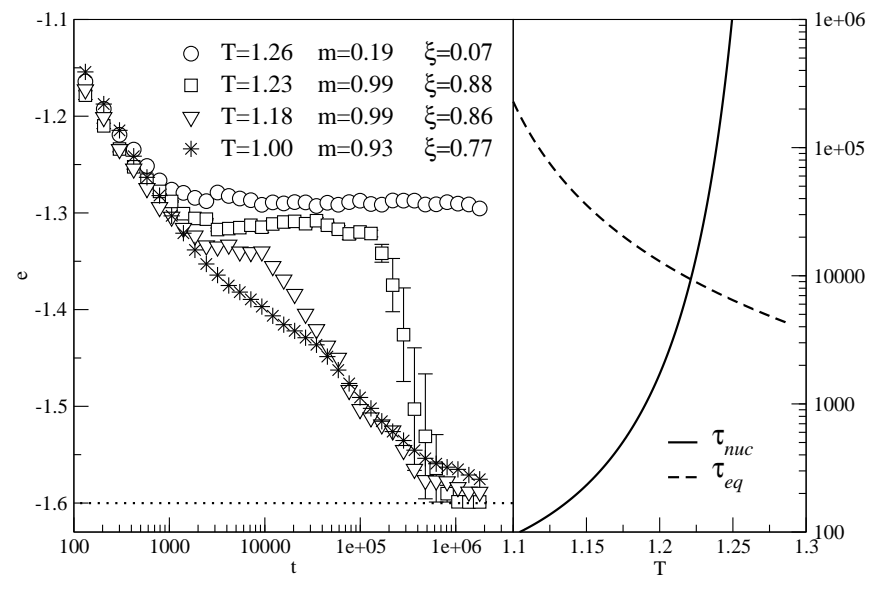

FIG. 2: Left: $e$ vs. $t$ at different temperatures, $L=100$. Error bars are only showed when larger than symbols size. The values of $m$ and $\xi$ in the legend are measured at the longest time for each temperature (see text). Right: crystal nucleation time and liquid equilibration time vs. temperature.

By imposing $\tau_{n u c}\left(T_{\mathrm{sp}}\right)=\tau_{e q}\left(T_{\mathrm{sp}}\right)$, we obtain the effective spinodal temperature $T_{\mathrm{sp}}$ marking the metastability limit. Fig. 2 (right) shows that $T_{\mathrm{sp}}=1.22$ [17]. We have $T_{\mathrm{sp}}>T_{s}$, and the Kauzmann paradox is thus avoided.

Below the metastability limit $T_{\mathrm{sp}}$ the only equilibrium phase is the crystal. However, a long-lived off-equilibrium glassy phase can still be formed: if we cool the system, it eventually remains stuck in an off-equilibrium state whose asymptotic energy is lower the slower the cooling rate $r$ (Fig. 3). All the configurations reached at $T=0$ are stable minima, indicating that activation is needed to grow the crystal. For very fast coolings these configurations are completely disordered, while at the slowest cooling rates they correspond to highly ordered polycrystals. Is there a qualitative difference among these asymptotic states? In other words, is it possible to sharply separate a bona fide glass from the polycrystal ?

To answer this question we introduce two direct measures of crystalline order. The first one is the normalized domain size $\xi$, obtained from the Fourier transform $G(k)$ of $G(r)=\left\langle s_{i} s_{i+r}\right\rangle . G(k)$ has a peak at $k_{0}=2 \pi / 5$, and we define $\xi$ as the inverse of the peak width, normalized by $L$. The second is the normalized crystal mass $m$, i.e. the total fraction of crystallized spins (defined as the number of spins down surrounded by 8 spins up, multiplied by 5) divided by $L^{2}$ [16]. Using $\xi$ and $m$ we see that crystallization has been achieved at the lowest three temperatures of Fig. 2. If we now plot the energy of the $T=0$ asymptotic configurations in a cooling experiment vs their crystalline mass and domain size, (Fig. 3 , inset), we find a continuous spectrum of states. Thus, in the CTLS there is structural continuity between highly disordered glassy minima and strongly ordered polycrystalline minima. The answer to the question above is therefore negative. Yet, had our slowest cooling rate been

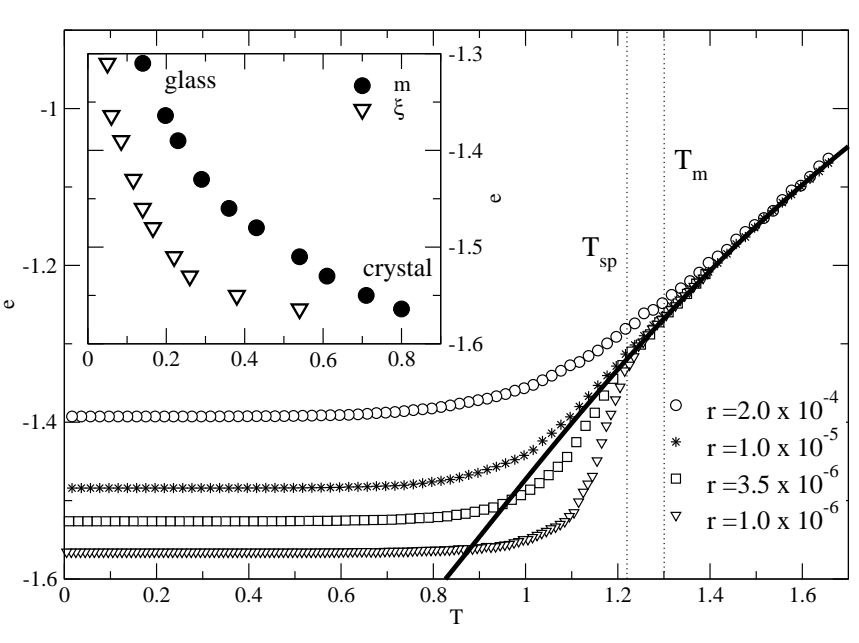

FIG. 3: $e$ as a function of $T$, at various cooling rates $r$. Full line: equilibrium liquid energy, eq. (2). Inset: $e$ vs $m$ and $\xi$ in the $T=0$ asymptotic states $\left(r \in\left[10^{-6}, 10^{-3}\right]\right) . L=100$.

$r=2 \times 10^{-4}$ we would only observe a disordered glass.

If not by structural difference, we might expect the dynamic behaviour of our "glass" to betray its nature of crystal-growth phase, by displaying a characteristic coarsening dynamics. In coarsening, excess energy over the ground state is concentrated in the interfaces among domains. This gives $e(t)-e_{\mathrm{GS}} \propto \xi(t)^{d-1} / \xi(t)^{d}=1 / \xi(t)$, for $L \xi \gg a$, where $a$ is the interfacial width. We do in fact find such a regime (Fig. Q1) for $\xi>0.2$. However, due to slow activated dynamics, the early regime, $L \xi \sim a$, can last for long, and in fact it is the only one that can be observed at the lowest temperatures. This regime, which we call bubbling, is characterized by a rapid increase of $m$ at roughly zero $\xi$ (Fig. 6 , inset), meaning that fast nucleation leads to rapid formation of many tiny crystal droplets. Only at longer times, when most of the system has crystallized, the domains grow at expense of each other and proper coarsening starts. For large systems we expect $m \sim 1-a / L \xi$, such that bubbling and coarsening regimes become well separated. Summarizing, at low temperatures, or for short experimental times, the coarsening regime is inaccessible, in the same way as, for fast cooling, polycrystals are not observed.

This behaviour has important consequences on the FDT violation pattern. The integrated response is given by $\chi\left(t, t_{w}\right)=\int_{t_{w}}^{t} d t^{\prime} R\left(t, t^{\prime}\right)$, where $R\left(t, t^{\prime}\right)=$ $\delta\langle s(t)\rangle / \delta h\left(t^{\prime}\right)$ and $h$ is the conjugate field to the spin. We can make a parametric plot of $\chi\left(t, t_{w}\right)$ vs the correlation $C\left(t, t_{w}\right)=\left\langle s(t) s\left(t_{w}\right)\right\rangle$. At equilibrium FDT holds and $\chi=\beta(1-C)$, while a departure from FDT is normally observed for late times if the system is out of equilibrium. This FDT violation is the standard tool to discriminate genuine glassy behaviour from simple domain growth. The relation $\chi=\beta X(1-C)$ can be used to express the late time FDT violation in off-equilibrium systems. For simple domain growth $X=0$, while in structural glasses 


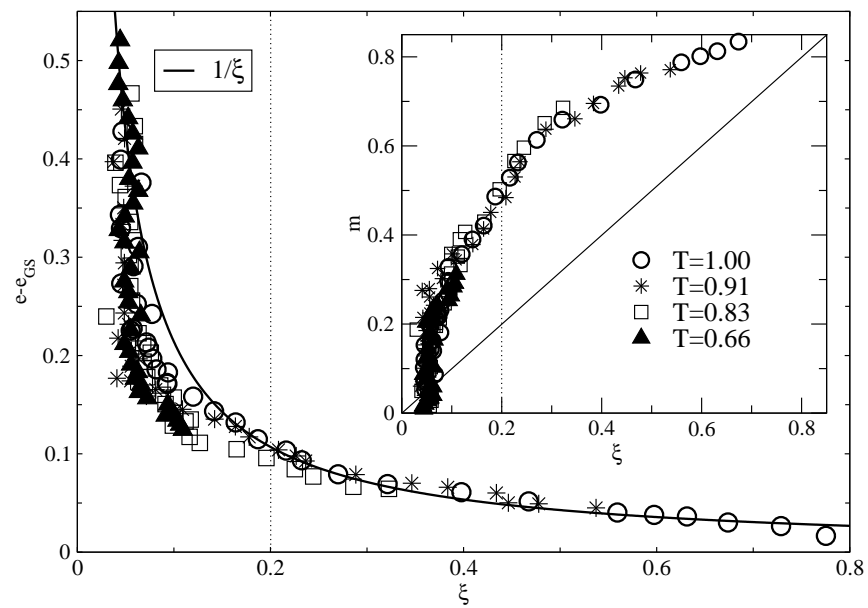

FIG. 4: Excess energy $e-e_{\mathrm{GS}}$ as a function of $\xi$. Full line: $1 / \xi$ fit of the data. Inset: $m(t)$ as a function of $\xi(t)$, parametrically in $t$, after a quench at various temperatures. All runs are $2 \cdot 10^{6}$ MCS long. $L=100$.

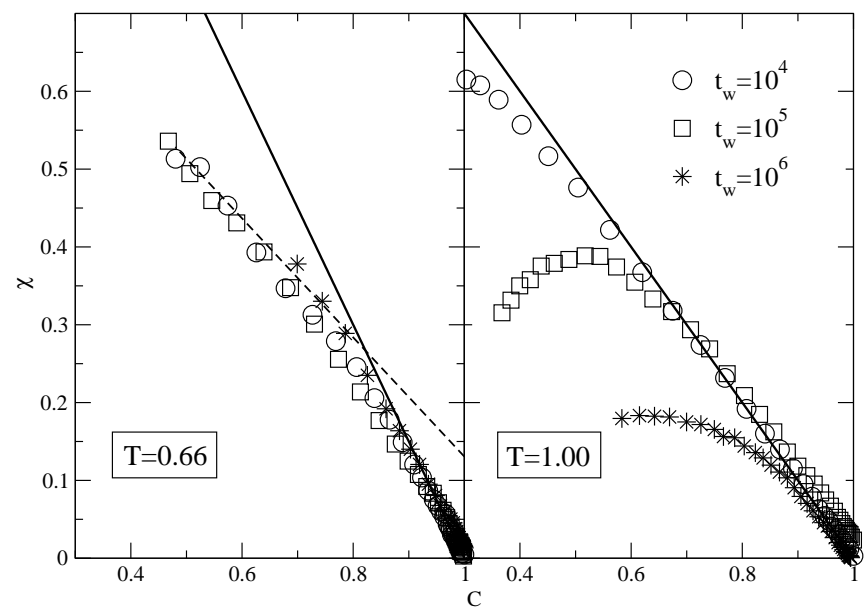

FIG. 5: Parametric plot of $\chi\left(t, t_{w}\right)$ vs $C\left(t, t_{w}\right)$, at various values of the waiting time $t_{w}$. Full lines represent the equilibrium relation $\chi=\beta(1-C)$. For $T=0.66$ the broken line is a linear fit for $C<0.8$. $L=500$.

it is observed $0<X<1$ [5, 6, 18]. We do expect a trivial FDT violation $(X=0)$ in the coarsening regime, but the situation may be more complicated in the earlier bubbling regime. From Fig. 4 we see that at $T=0.66$ the system remains in the bubbling regime up to the largest time. At the same temperature we find an FDT violation very similar to structural glasses (Fig. [): for $C$ smaller than a given breaking point the slope changes, giving rise to a constant nonzero value of $X$. Moreover, the pattern basically does not change within two orders of magnitude in the waiting time $t_{w}$. At $T=0.66$ we can thus define an effective temperature $T_{\text {eff }}=T / X=1.32$. On the other hand, for $T=1$ as $t_{w}$ increases we see a crossover from a nontrivial FDT violation $(X \neq 0)$, to a seemingly trivial pattern $(X \sim 0)$. This is consistent with the fact that at $T=1$ the coarsening regime is reached within our exper- imental time (Fig. đ). Yet, once again, had our longest experimental time been too short to enter the coarsening regime, we would only observe a glassy FDT violation.

In this Letter we presented a model where the metastability limit can be either observed directly as a loss of stability of the supercooled liquid, or indirectly, thanks to the formation of polycrystalline asymptotic states in slow cooling experiments, and thanks to trivial FDT violation in the late coarsening regime. However, in order to be effective the indirect methods need as long an experimental time as the direct method. For shorter time scales the present model is compatible with ordinary fragile glasses.

We acknowledge many important discussions with P. Debenedetti, H. Horner, V. Martin-Mayor, G. Parisi, F. Ricci-Tersenghi, F. Sciortino, D. Sherrington and P. Verrocchio. AC thanks in particular M. A. Moore.

[1] W. Kauzmann, Chem. Rev. 43, 219 (1948).

[2] G. Adam and J.H. Gibbs, J. Chem. Phys. 43, 139 (1965).

[3] A. Crisanti and H.J. Sommers, J. Phys. I 5, 806 (1995).

[4] M. Mezard and G. Parisi, J. Phys.: Condens. Matter 11, A157 (1999) and references therein; F. Sciortino, W. Kob and P. Tartaglia, Phys. Rev. Lett. 83, 3214 (1999).

[5] L.F. Cugliandolo, J. Kurchan and L. Peliti, Phys. Rev. E 55, 3898 (1997); A. Barrat, Phys. Rev. E 57 (1998) 3629 .

[6] W. Kob and J.L. Barrat, Phys. Rev. Lett. 78, 4581 (1997); G. Parisi, Phys. Rev. Lett. 79, 3660 (1997); T. S. Grigera and N. E. Israeloff, Phys. Rev. Lett. 87, 085502 (2001).

[7] A similar interpretation of the so-called glacial phase in the fragile glass-former TTP, can be found in A. Hédoux et al., J. Chem. Phys. 116, 9374 (2002).

[8] Data obtained at $L=500$ and $L=100$ are quantitatively consistent.

[9] T. Grigera and N.E. Israeloff, Phil. Mag. B 82, 313 (2002).

[10] J. D. Shore, M. Holzer and J. P. Sethna, Phys. Rev. B 46, 11376 (1992);

[11] A. Lipowski, J. Phys. A 30,7365 (1997); A. Lipowski and D. A. Johnston, Phys. Rev. E 61, 6375 (2000); M. R. Swift, H. Bokil, R. D. M. Travasso and A. Bray, Phys. Rev. B 62, 11494 (2000).

[12] A. Buhot and J.P. Garrahan, Phys. Rev. Lett. 88, 225702 (2002).

[13] G. Biroli and M. Mezard, Phys. Rev. Lett. 88, 025501 (2002).

[14] C.A. Angell, J. Phys. Chem. Solids 49, 1924 (1988).

[15] F.F. Abraham, Homogeneous Nucleation Theory (Academic, New York, 1974).

[16] Both $\xi$ and $m$ have an effective zero given by their value in a random configuration, $\xi_{0}=0.05, m_{0}=0.01$.

[17] A discussion of the connection between $T_{\mathrm{sp}}$ and $T_{c}$ in a similar model can be found in M.R. Swift et al. [11].

[18] For an analysis of FDT violation in preasymptotic simple coarsening see, L. Berthier, J.-L. Barrat, J. Kurchan, Eur. Phys. J. B 11, 635 (1999). 